\title{
Analysis of Efficient Terahertz cavity phase matching Difference Frequency Generation in a GaAs Crystal sheet
}

\author{
Hui-Hui Xu ${ }^{1, a}$, Zhi-Ming Rao ${ }^{1, b}$ and Fang-Sen $X^{1, c^{*}}{ }^{*}$ \\ ${ }^{1}$ School of Physics, Communication and Electronics, Jiangxi Normal University, nanchang, Jiangxi, \\ 330022, CHINA \\ a506610113@qq.com, raozm24@163.com, ${ }^{\mathrm{c}}$ 121425353@qq.com \\ * corresponding author : 121425353@qq.com
}

Keywords: terahertz, cavity phase matching, difference frequency generation, GaAs crystal sheet.

Abstract. In this paper explore the terahertz (THz) wave generation based on cavity phase matching (CPM) difference frequency generation (DFG) in a GaAs crystal sheet. This scheme applying cavity phase matching (CPM) is proposed. The wave propagation of the higher frequency pump wave is parallel to the wave propagation of the lower frequency pump wave, and both of them are perpendicular to the cavity length in a GaAs crystal sheet. The $\mathrm{THz}$ wave is emitted the nonlinear crystal. We calculated the optimized cavity length in a GaAs crystal sheet at the range of $100-1000 \mu \mathrm{m}$ $(0.3-3 \mathrm{THz})$. Our estimates illustrate that $\mathrm{THz}$ wave output corresponds to the power conversion efficiency of 50 times in CPM-DFG for in DFG. The result is shown that this scheme is efficient to obtain $\mathrm{THz}$ wave.

\section{Introduction}

Terahertz (THz) sources can be used in many applications in environment detections, security inspections, biomedical diagnostics, and so on [1-4]. Difference frequency generation (DFG) was designed to satisfy the wave vector phase matching condition in nonlinear crystal, and DFG is one of the effective ways to produce high-power, narrow-band coherent $\mathrm{THz}$ sources in nonlinear crystals, such as periodically poled $\mathrm{MgO}: \mathrm{LiNbO}_{3}$ [5], periodically poled $\mathrm{LiNbO}_{3}$ (PPLN) [6], and periodically-inverted GaAs [7]. The conversion efficiency is dependent on the signal and pump beam overlap, focusing conditions, crystal temperature, crystal length, and crystal absorption coefficients in the $\mathrm{THz}$ wave region. However, nonlinear optical crystals have high absorption coefficients in the $\mathrm{THz}$ wave region, which limited the interaction length in crystals and lowered the output $\mathrm{THz}$ wave power. The scheme of cavity phase matching using has been proposed $[8,9]$ and demonstrated [10] to solve nonlinear optical crystals high absorption coefficients. In this scheme, the $\mathrm{THz}$ wave is generated in cavity phase matching. CPM-DFG makes it possible to avoid $\mathrm{THz}$ wave strongly absorbing crystals decay and pump wave achieves 10 times in a GaAs crystal sheet.

\section{Theory of scheme and discussion}

GaAs crystal has a large nonlinear coefficient, a widely transparency range, and a high damage threshold. According to the Manley-Rowe relation, the maximum conversion efficiency can be improved by one order of magnitude when using mid-infrared laser with longer wavelength running at $10 \mu \mathrm{m}$. Taking into account the maturity of mid-infrared laser technology and the development of technology of periodically-inverted crystals, it will be an effective way to generate $\mathrm{THz}$ wave based on CPM-DFG. However, the theory generation of the efficient $\mathrm{THz}$ wave in a crystal sheet cavity phase matching pumped by wavelength running at $10 \mu \mathrm{m}$ laser has not been detail reported.

In this letter, explore the terahertz $(\mathrm{THz})$ wave generation based on cavity phase matching $(\mathrm{CPM})$ difference frequency generation (DFG) in a GaAs crystal sheet. It is found that the Zinc blende semiconductor GaAs is an efficient $\mathrm{THz}$ frequency generator owing to its large second-order nonlinear coefficient $\left(\mathrm{d}_{36}(10.6 \mu \mathrm{m})=83 \mathrm{pm} / \mathrm{V}\right)[11]$. Our scheme is cavity phase matching $(\mathrm{CPM})$. The optimized cavity length in a GaAs crystal sheet and the power conversion efficiency were calculated in this paper. 
The geometry principle of phase matching is as shown. Here we assume that the wave propagation of the higher frequency pump wave (at frequency $\omega_{l}$, wavelength $\lambda_{l}$ ) is parallel to the wave propagation of the lower frequency pump wave (at frequency $\omega_{2}$, wavelength $\lambda_{2}$ ), and both of them are perpendicular to the domain wall of the nonlinear crystal. The THz wave (at frequency $\omega_{T}$, wavelength $\lambda_{T}$ ) is emitted the nonlinear crystal.

The damping of the THz wave is minimized due to the short pass length in the nonlinear crystal. The optimized cavity length in a GaAs crystal sheet $\Lambda$ can be calculated by using the vector phase matching condition and energy conservation:

$$
k_{1}-k_{2}-k_{T}=\frac{\pi}{\Lambda},
$$

1)

$$
\frac{1}{\lambda_{T}}=\frac{1}{\lambda_{1}}-\frac{1}{\lambda_{2}},
$$

\section{2)}

where, $k_{i}=2 \pi n_{i} / \lambda_{i}$ and $n_{i}(\mathrm{i}=1,2, \mathrm{~T})$ denote high frequency pump, low frequency pump and THz wave vector and refractive indices, respectively. The grating period $\Lambda$ can be deduced from equations (1) and (2)

$$
\Lambda=\frac{\lambda_{1} \lambda_{T}}{2\left(n_{1} \lambda_{T}-n_{T} \lambda_{1}-\lambda_{T} n_{2}+\lambda_{1} n_{2}\right)},
$$

This scheme can produce THz wave. For $\lambda_{I}=9.552 \mu \mathrm{m}$, we calculated optimized cavity length in a GaAs crystal sheet $\Lambda$ by using the Sellmeier equation of GaAs from Ref. [12] and Eq. (3). The Sellmeier equation of GaAs is given by

$$
n^{2}(\lambda)=b+\frac{g_{1}}{b_{1}^{-2}-\lambda^{-2}}+\frac{g_{2}}{b_{2}^{-2}-\lambda^{-2}}+\frac{g_{3}}{b_{3}^{-2}-\lambda^{-2}},
$$

where, $\lambda$ is the pump or Thz laser wavelength, $b=5.372514, b_{1}(\mu \mathrm{m})=0.4431307+0.000050564 \Delta \mathrm{T}$, $\mathrm{b}_{2}(\mu \mathrm{m})=0.8746453+0.0001913 \Delta \mathrm{T}-4.882 \times 10^{-7} \Delta \mathrm{T}^{2}, \quad \mathrm{~b}_{3}(\mu \mathrm{m})=36.9166-0.011622 \Delta \mathrm{T}, \quad \Delta \mathrm{T} \quad$ is the deviation from the reference room temperature $22 \square, \quad \mathrm{g}_{1}=27.83972$, $\mathrm{g}_{2}=0.031764+4.350 \times 10^{-5} \Delta \mathrm{T}+4.664 \times 10^{-7} \Delta \mathrm{T}^{2}$, and $\mathrm{g}_{3}=0.00143436$.

The relation of the optimized cavity length in a GaAs crystal sheet $\Lambda$ at room temperature and the THz wavelength are shown in Fig. 1 from Eqs.(3) and (4). It can be seen that $\Lambda$ is increased from 0.16 to $1.96 \mathrm{~mm}$ when $\mathrm{THz}$ wavelength is increased from 100 to $1000 \mu \mathrm{m}$ in the CPM scheme. Needing the optimized cavity length in a GaAs crystal sheet is a $0.58 \mathrm{~mm}$ in the CPM scheme to generate a frequency of 1

$\mathrm{THz} \quad(300 \mu \mathrm{m})$ wave.

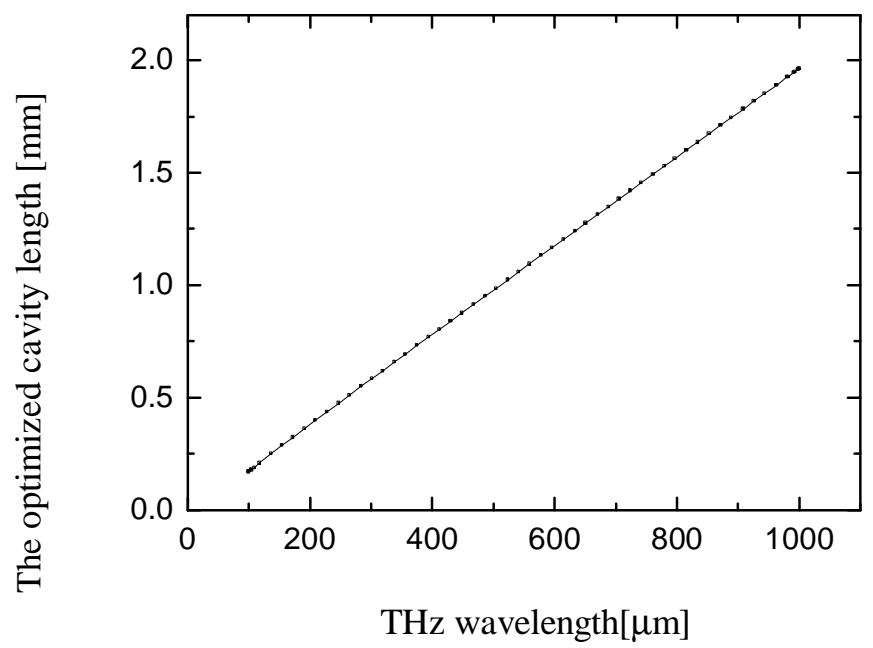

Fig.1. The optimized cavity length versus the generated THz wavelength with a GaAs crystal sheet 
To evaluate the performances for using crystals as THz-wave converters, all the interactions can be phase matched in DFG, and THz power conversion efficiency $\eta$ in the plane-wave fixed-field can be given by [13]

$$
\eta=\frac{P_{T}}{P_{1}}=\frac{8 \pi^{2} d_{e f f}^{2} L^{2} P_{2}}{\varepsilon_{0} c n_{1} n_{2} n_{T} \lambda_{T}^{2} A} e^{-\alpha_{T} L},
$$

where, $L$ is the crystal length $0.058 \mathrm{~cm}$, the pump intensity $P_{2}=10 \mathrm{MW}$, dielectric constant of vacuum $\varepsilon_{0}=8.854187817 \times 10^{-12} \mathrm{~F} / \mathrm{m}$, light speed of vacuum $\mathrm{c}=3 \times 10^{8} \mathrm{~m} / \mathrm{s}$, and the pump area $A=0.1 \mathrm{~mm}^{2}$. Zinc blende semiconductor GaAs THz absorption coefficient $\alpha_{\mathrm{T}}$ is $0.5-4.5 \mathrm{~cm}^{-1}$ at $1-3 \mathrm{THz}$ [7]. For DFG process, when THz-wave $\lambda_{T}=300 \mu \mathrm{m}(1 \mathrm{THz})$ from Eq. (5), which corresponds to the power conversion efficiency is of $\sim 0.000001 \%$.

In this CPM-DFG scheme, to evaluate the performances for using crystals as THz-wave converters, assume that the pump wave achieves 10 times in a GaAs crystal sheet. THz-wave power conversion efficiency $\eta$ neglecting pump depletion in the plane-wave fixed-field can be given by

$$
\eta=\frac{P_{T}}{P_{1}}=\frac{3200 d_{\text {eff }}^{2} L^{2} P_{2}}{\varepsilon_{0} c n_{1} n_{2} n_{T} \lambda_{T}^{2} A},
$$

where, $b=0.3 \mathrm{~mm}$ is the crystal thickness, and the overlap factor $F=1$. For the CQPM scheme, at THz-wave $\lambda_{T}=100 \mu \mathrm{m}$ (3THz) from Eq.(6), which corresponds to the power conversion efficiency of $\sim 0.00005 \%$. However, the power conversion efficiency of THz-wave at $3 \mathrm{THz}$ is only $0.000001 \%$ in DFG. In CPM-DEG, the power conversion efficiency of THz-wave is far more than that of in DFG, which is due to the CPM-DEG pump wave achieves 10 times in a GaAs crystal sheet.

\section{Summary}

In conclusion, we have investigated THz generation from the optimized cavity length in a GaAs crystal sheet with wavelength running at $10 \mu \mathrm{m}$, and scheme of phase matching is CPM pattern. Using CPM-DEG the pump wave achieves 10 times in a GaAs crystal sheet, and the power conversion efficiency is $0.00005 \%$ at a frequency of $1 \mathrm{THz}$, since the pump beam is achieve 10 times in a GaAs crystal sheet. We calculated the optimized cavity length in a GaAs crystal sheet at $\mathrm{THz}$ wavelength from 100 to $1000 \mu \mathrm{m}(0.3-3 \mathrm{THz})$. It is shown that the optimized cavity length in a GaAs crystal sheet is efficient to obtain high power conversion efficiency of $\mathrm{THz}$ wave generation with mid-infrared laser by using CPM-DEG.

\section{Acknowledgement}

This work is supported by the science and technology plan of jiangxi province (20151BBE50089) and by the doctoral Fund of Jiangxi Normal University (6398).

\section{References}

[1] Y.M. Bahk, H.R. Park, K.J, et al: Ahn: Phys. Rev. Lett. Vol.106 (2011), 013902.

[2] C.A. Schmuttenmaer: Chem. Rev. Vol.104(2004), p.1759-1779.

[3] X. Xie, J. Dai, Zhang. X.C: Phys. Rev. Lett. Vol.96 (2006), 075505.

[4] M. B. Johnson, D.M. Whittaker, et al: Linfield: Phys. Rev. B. Vol. 65(2002), 165301.

[5] D. Molter, M .Theuer, and R .Beigang: Opt. Express. Vol.17(2009), p.6623-6628

[6] Y.S. Lee, T. Meade, et al: Appl. Phys. Lett. Vol. 76(2000), p.2505-2507.

[7] K.L.Vodopyanov, M.M. Fejer, X. Yu, J.S. Harris, Y.S. Lee, W.C .Hurlbut, V.G. Kozlov, D. Bliss, and C. Lynch: Appl. Phys. Lett. Vol. 89(2006), 141119.

[8]Armstrong J A, Blombergen N1962, Physics Review, 127(6):1918-1939. 
[9]Haidar R, Forget N, and Rosencher E: 2003,Quantum Electron, 39 $\square: 569-575$.

[10] Xie Z D, Lv X J,Liu Y H, et al.2011,Rhysical Review Letters, 106(8):083901.

[11] D. N. Nikogosyan: Nonlinear Optical Crystals: A Complete Survey (New York: Springer) 2005, p208

[12] T. Skauli, P.S. Kuo, K.L. Vodopyanov, T.J. Pinguet, O. Levi, L.A. Eyres, J.S. Harris, B. Gerard, L. Becouarn, and E. Lallier: J. Appl. Phys.Vol.94(2003), p.6447.

[13] V. G. Dmitriev: Handbook of Nonlinear Optical Crystals (Springer publications, Berlin 1999) p50 